\title{
How long can a graph be kept planar?
}

\author{
V. Anuradha Chinmay Jain Jack Snoeyink Tibor Szabó \\ IIT Bombay $\quad$ IIT Bombay $\quad$ UNC Chapel Hill $\quad$ ETH Zurich
}

Submitted: Nov 2, 2007; Accepted: Apr 25, 2008; Published: May 5, 2008

Mathematics Subject Classification: 91A24

\begin{abstract}
The graph (non-)planarity game is played on the complete graph $K_{n}$ between an Enforcer and an Avoider, each of whom take one edge per round. The game ends when the edges chosen by Avoider form a non-planar subgraph. We show that Avoider can play for $3 n-26$ turns, improving the previous bound of $3 n-28 \sqrt{n}$.
\end{abstract}

\section{Introduction}

The (non-)planarity game is a positional game between two players, Avoider and Enforcer, played on the edges of the complete graph on $n$ vertices. The game consists of a sequence of rounds, in each round Enforcer occupies one previously unoccupied edge of $K_{n}$, followed by Avoider occupying one. For clarity, let us assume that Enforcer starts the game, but this is not crucial, our result applies even if Avoider does. Avoider seeks to avoid creating a nonplanar graph - he aims that his chosen edges should have a planar embedding. Enforcer chooses edges that makes Avoider's task more difficult - she tries to force Avoider's graph to become non-planar.

Independent of her strategy, Enforcer inevitably succeeds by round $3 n-5$, when Avoider's graph has simply too many edges to be planar. József Balogh [1] gave a simple strategy for Enforcer that makes Avoider fail already by turn $3 n-7$. : Once Avoider choses his first edge $a b$, Enforcer pairs $(v a, v b)$ for all vertices $v \neq a, b$, and whenever Avoider chooses one edge of a pair, Enforcer chooses the other. This ensures that $a b$ cannot participate in any triangle, so contracting $a b$ removes a single edge from Avoider's graph and leaves the result planar. Thus, Avoider has at most $3(n-1)-6+1=3 n-8$ edges. From the other side, Avoider can fix an arbitrary triangulation on the $n$ vertices and make sure that he selects at least half of its edges during his turns; thus delaying his defeat for at least $\frac{3}{2}(n-6)$ rounds.

Hefetz al. [7] gave a strategy for Avoider that allowed him to keep his graph planar for at least $3 n-28 \sqrt{n}$ turns. In this paper we provide a better strategy for Avoider and obtain a result that is best possible up to an additive constant. 
Theorem 1. In the planar graph game on $n$ vertices, Avoider can play at least $3 n-26$ edges while keeping his graph planar.

Motivation and History Positional games consist of a board, which is a finite set $X$, and a collection $\mathcal{F} \subset 2^{X}$ of winning sets, which is usually assumed to be a monotone (increasing) family. The board is often the vertices or edges of a graph; winning sets may be spanning trees, perfect matchings, $s-t$ connected paths, or Hamiltonian cycles. In a Maker/Breaker positional game, the two players take turns choosing elements of $X$. Maker wins by choosing all elements of some winning set, otherwise Breaker wins. The work of Erdős and Selfridge [5] established a general condition for Breaker to win, and stimulated much other work, notably that of Beck; see his book [3] for an excellent treatise of positional games in a broad sense.

Hefetz et al. [6] suggest that Avoider/Enforcer games, the misère version of Maker/ Breaker games, in which Avoider's goal is to avoid completely occupying any of the members of $\mathcal{F}$, is a similarly natural setup. One such scenario is when a Maker/Breaker game is played on a monotone decreasing family $\mathcal{H}$, for example the family of planar graphs. This is then equivalent to playing an Avoider/Enforcer game on the complement increasing hypergraph $2^{E\left(K_{n}\right)} \backslash \mathcal{H}$. Our targeted game of interest, the non-planarity game, also arose this way. We denote by $\mathcal{N} \mathcal{P}_{n} \subseteq 2^{E\left(K_{n}\right)}$ the family of those edge sets on $n$ vertices that determine a non-planar graph.

Maker/Breaker and Avoider/Enforcer games are perfect information games without the possibility of a draw, i.e. exactly one of the players has a winning strategy (and his identity is determined by the family $\mathcal{F}$ alone). For natural, well-studied graph games it often so happens that the (in some sense) "easy" winner is Maker or Enforcer, respectively. As discussed in the introduction, our game of interest, the non-planarity game $\mathcal{N} \mathcal{P}$ is one such instance. There are several ways suggested in the literature to "even out" such an adventage, which then would "measure" how easy such a win is. One approach, suggested by Chvátal and Erdős[4], to give Breaker a bias, i.e., allow him to take $b>1$ edges in each turn instead of just one. Another possibility, suggested in [8], is to randomly reduce the board and search for the threshold probability at which Maker's win turns into Breaker's win. A third way, introduced in [7], measures how fast can Maker (or Enforcer) win. The parameters $\tau_{M}(\mathcal{F})$ and $\tau_{E}(\mathcal{F})$ are defined as the smallest number of rounds Maker and Enforcer can win their respective games on $\mathcal{F}$. The parameters are defined to be $\infty$ if Breaker or Enforcer wins, respectively. In [7, 2] these parameters are studied in depth, their value is estimated and occasionally determined for several natural graph games, for example the games involving spanning trees, perfect matchings, Hamilton cycles, $k$ colorability, or planarity.

For large $n$, the Maker/Breaker parameter $\tau_{M}\left(\mathcal{N} \mathcal{P}_{n}\right)$ is easily seen to be bounded by a constant independent of $n$, since Maker can create a $K_{5}$ in a constant number of moves on a large enough board.

In the present paper we investigate the Avoider/Enforcer parameter $\tau_{E}\left(\mathcal{N} \mathcal{P}_{n}\right)$ and obtain a result tight up to an additive constant. 


\section{Avoider Strategy}

In this section we detail Avoider's strategy, which we can summarize as follows: Avoider initially designates four anchor vertices in two pairs, $a_{0}, a_{1}$ and $b_{0}, b_{1}$. In his turns, Avoider adds an edge to connect a remaining vertex to one anchor from each pair, or else to play a permutation game that builds paths joining vertices that are connected to the same pair of anchors. The resulting graph will have a planar embedding; as in Figure 1, Avoider can embed a $K_{4}$ for the anchors, then lay out the remaining vertices and their paths near the edges of $K_{4}$ that join the corresponding anchors.

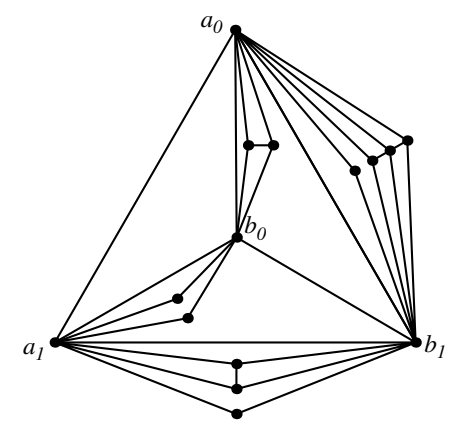

Figure 1: Planar layout of Avoider's edges

First, however, we define some more terminology and notation, so that we can state some invariant properties of Avoider's strategy.

Let $V$ be the set of $n-4$ non-anchor vertices. Avoider will connect each $v \in V$ to exactly two anchors $a_{i}$ and $b_{j}$, for some $i, j \in\{0,1\}$ - in this case we say that $v$ is pinned to $i j$. We write $\mathrm{u} \prec \mathrm{v}$ if $\mathrm{u}$ is pinned to two anchors before $\mathrm{v}$ is pinned to two anchors.

After $v$ is pinned to $i j$, Avoider may choose edges that join $v$ to at most two other vertices that have also been pinned to $i j$. We say that a vertex is active in ij if its Avoider degree is two or three, that is, it is pinned to $i j$, but not yet connected to two other neighbors.

A key idea in Avoider's strategy is to try to play an edge incident to Enforcer's just chosen edge, $e$, unless it is already clear that the endpoints of $e$ can never be active in the same $i j$. Specifically, if Enforcer's chosen edge $e$ has an unpinned endpoint, Avoider will connect it to an anchor. On the other hand, if both endpoints are pinned to the same $i j$, Avoider will try to play an incident edge among the vertices currently active in $i j$.

In fact, this may not always be possible: An edge $e=u v$ that Enforcer chooses is special if and only if vertices $u$ and $v$ are both active in $i j$ (for some $i, j \in\{0,1\}$ ) during her turn, and Avoider has no edge from $u$ or $v$ to another vertex active in $i j$ to choose in his next turn. In case $u \succ v$, Avoider directs Enforcer's special edge from $u$ to $v$.

Now, we are ready to give the components of Avoider's strategy in responding to an Enforcer's choice of an edge.

1. Anchor Pairing Strategy: Enforcer chooses an edge between two anchors. 
Then Avoider answers with an edge paired with Enforcer's choice in the following (somewhat arbitrary) list: $\left(a_{0} a_{1}, a_{0} b_{0}\right),\left(a_{1} b_{0}, a_{1} b_{1}\right),\left(a_{0} b_{1}, b_{0} b_{1}\right)$.

2. Pinning Strategy: Enforcer chooses an edge $e$ that is incident to at least one unpinned vertex.

Then Avoider connects an unpinned endpoint $u$ of $e$ to an anchor.

If $e$ was incident to an anchor, say $e=u a_{i}$, then Avoider's first preference is to take the edge between $u$ and the pair of the anchor, namely $u a_{1-i}$. Otherwise, that is, if $u a_{1-i}$ is already taken by Avoider or $e$ is not incident to an anchor, then Avoider connects $u$ to $a_{0}$ or $b_{0}$, whichever is available (selecting arbitrarily if both $u a_{0}$ and $u b_{0}$ are free). Observe that by the preference list in the Pinning Strategy, for any unpinned vertex $u$, one of $u a_{0}$ and $u b_{0}$ must be free.

3. Permutation Game Strategy: Enforcer chooses an edge $u v$ incident to two active vertices in $i j$.

Then if some edge exists from $u$ or $v$ to another active vertex in $i j$, Avoider chooses such an edge.

Otherwise, Enforcer's edge $u v$ is special, and Avoider chooses an edge between two anchors, or connects any unpinned vertex to $a_{0}$ or $b_{0}$, or joins two vertices active in any $i^{\prime} j^{\prime}$, (as in 1., 2., and 3.)

4. Enforcer chooses any other edge (such as an edge incident to a pinned but non-active vertex, or an edge joining vertices that are not pinned to the same $i j$ ).

Then, again, Avoider chooses an edge between two anchors, or connects any unpinned vertex to $a_{0}$ or $b_{0}$, or joins two vertices active in any $i^{\prime} j^{\prime}$.

\section{Analysis}

In this section we will prove that Avoider's strategy ensures that at least $3 n-26$ rounds are played before Avoider's graph becomes non-planar. For convenience, the moment when Avoider has no more edges he can take according to rules 1., 2., 3., or 4. we end the game, even though Avoider's graph is still planar.

We call an edge of Enforcer normal if it connects two non-anchor vertices and right after its selection Avoider selected an edge incident to it (according to 2. or 3.) Observe that at the end of the game every Enforcer edge that connects two vertices active in the same $i j$ will be either normal or special.

The first lemma states that Avoider will select at least half of the edges incident to anchors.

Lemma 2. Avoider chooses at least three of the six edges between anchors, and guarantees that every non-anchor vertex $v \in V$ becomes pinned to some $i j$, with $i, j \in\{0,1\}$. 
Proof. Since each edge incident to an anchor is in exactly one pair of the Anchor Pairing Strategy and the Pinning Strategy, Avoider can guarantee to obtain one edge from each pair, no matter what Enforcer chooses.

To analyze the permutation strategy among vertices active in $i j$, we first bound the number of special Enforcer edges.

Lemma 3. The special edges in the graph induced by the vertices active in ij form a directed forest. Thus, between $p$ vertices active in ij there can be at most $p-1$ special edges.

Proof. We claim that a vertex $u$ has at most one special edge leaving it. Suppose that Enforcer chooses two edges, first $u v$ and later $u w$, with $u \succ v$ and $u \succ w$. Then $u v$ cannot be special because $u w$ was available with $w$ already active in $i j$ in the turn that Enforcer chose $u v$. Thus, the graph of special edges is acyclic and the bound follows.

Due to our preference for anchoring to $a_{0}$ and $b_{0}$, we can establish a lemma that bounds the number of normal Enforcer edges as a function of $i+j$.

One more bit of notation: the vertices pinned to ij can be partitioned by Avoider degree so that there are $p_{k}$ with Avoider degree $k$, for $k=2,3$, or 4 . Of these, $r=p_{2}+p_{3}$ are active in $i j$.

Lemma 4. Let $R$ be the set of vertices active in ij with $|R|=r$. Then the number of normal Enforcer edges in $R$ is at most $(2-(i+j)) r+p_{3}$.

Proof. According to the definition, for every Enforcer edge that is normal, Avoider creates an adjacent edge. Hence we can use Avoider degrees to bound the number of normal Enforcer edges in $R$, which would give at most $2 p_{2}+3 p_{3}=2 r+p_{3}$ edges. This can be sharpened for $i j \neq 00$ because edges $v a_{1}$ or $v b_{1}$, for some non-anchor vertex $v$, are selected by Avoider only as an answer to Enforcer selecting $v a_{0}$ or $v b_{0}$, respectively. In other words, these Avoider edges are not answers to a normal Enforcer edge. Thus, within vertices pinned to $i j$, we have $(2-(i+j)) r+p_{3}$ normal Enforcer edges.

Lemma 5. Let $P$ be the set of all vertices pinned to $i j$, so that $|P|=p_{2}+p_{3}+p_{4}$. Avoider will be able to choose at least $|P|-6-i \cdot j+2(i+j)$ edges in the subgraph induced by $P$.

Proof. The number of edges that Avoider chooses within $P$ can be calculated from the degrees as $p_{4}+p_{3} / 2=|P|-\left(p_{3} / 2+p_{2}\right)$. Thus, we maximize $\left(p_{3} / 2+p_{2}\right)$ subject to the constraint that Avoider has no edge to choose within $P$ (i.e., all possible answers are already picked up Enforcer).

Observe that every Enforcer edge connecting two vertices active in $i j$ is either normal or special.

In a permutation game with $r=p_{2}+p_{3}$ vertices active in $i j$, there are $\left(\begin{array}{l}r \\ 2\end{array}\right)$ possible edges, of which Enforcer has at most $r-1$ special edges (Lemma 3), and at most (2$(i+j)) r+p_{3}$ normal edges (Lemma 4). Avoider must avoid closing paths, which rules out $p_{3} / 2$ additional pairs, but he can choose any remaining edge between active vertices. 
The constraint that Avoider has no edge to choose gives these equivalent inequalities involving $p_{3}$ and $p_{2}$ :

$$
\begin{aligned}
\left(\begin{array}{l}
r \\
2
\end{array}\right) & \leq(2-(i+j)) r+p_{3}+(r-1)+\frac{p_{3}}{2} \\
r(r-1) & \leq(6-2(i+j)) r+3 p_{3}-2 \\
r-1 & \leq(6-2(i+j))+3 p_{3} / r-2 / r \\
p_{2}+p_{3} / 2 & \leq 7-2(i+j)-p_{3} / 2+3 p_{3} / r-2 / r .
\end{aligned}
$$

Since path endpoints come in pairs, $p_{3}$ is even and the left-hand side of the final inequality is an integer. When $r \geq 6$ or $p_{3}=0$, the expression $-p_{3} / 2+3 p_{3} / r-2 / r$ is negative, and the right side can be reduced to $\leq 6-2(i+j)$. For $p_{3}=2$ and $p_{3}=4$, we can thus assume $p_{2} \leq 3$ and $p_{2} \leq 1$, respectively. Only when $p_{3}=2, p_{2}=2$ or $p_{3}=4, p_{2}=1$ can equality be obtained in $p_{2}+p_{3} / 2 \leq 7-2(i+j)$, and then only in the case that $i=1$ and $j=1$. Thus we may write $p_{2}+p_{3} / 2 \leq 6+i \cdot j-2(i+j)$, which gives the expression stated in the lemma.

Now, we can establish Theorem 1: in the (non-)planarity game on $n$ vertices, Avoider can play at least $3 n-26$ edges while keeping his graph planar.

Proof. of Theorem 1. By Lemma 2, Avoider chooses at least 3 edges between anchors, and exactly $2(n-4)$ edges that pin each of the non-anchor vertices. At the end of the game, if there are $n_{i j}$ vertices pinned at $i j$, we have $\sum_{i, j \in\{0,1\}} n_{i j}=n-4$. By Lemma 5 , the number of edges that Avoider chooses in all four permutation games is

$$
\sum_{i, j \in\{0,1\}} n_{i j}-6-i \cdot j+2(i+j)=n-4-24-1+8=n-21 .
$$

Thus, the total number of edges chosen by Avoider is $3+2(n-4)+n-21=3 n-26$.

Figure 1 illustrates that Avoider's edges have a planar embedding, which concludes the proof.

\section{Open problems}

As it was pointed out by some of the participants of the 2007 GREMO workshop, the pairing strategy idea of Balogh, mentioned in the introduction, can be carried out on two edges, which guarantees an Enforcer win by round $3 n-8$. However, there remains a gap between the upper and lower bounds, which could be narrowed if we could show that the number of special Enforcer edges is smaller.

\section{Acknowledgment}

We thank all the participants of the 2007 GREMO workshop on open problems, especially Emo Welzl, for discussions on this and other problems. Work of Dr Snoeyink was partially supported by NSF 0429901. 


\section{References}

[1] J. Balogh, private communication.

[2] J. Balogh, R. Martin, On Avoider-Enforcer games, submitted.

http://orion.math.iastate.edu/rymartin/papers/avoidHsubmit0816.pdf

[3] J. Beck, Combinatorial games: Tic-Tac-Toe Theory, Cambridge University Press, 2008.

[4] V. Chvátal, P. Erdős, Biased positional games, Annals of Discrete Mathematics, 2 (1978), 221-228.

[5] P. Erdős and J. Selfridge, On a combinatorial game, J. Combinatorial Theory, Ser. A 14 (1973) 298-301.

[6] D. Hefetz, M. Krivelevich and T. Szabó, Avoider-Enforcer games, J. Combinatorial Theory, Ser. A. 114 (2007) 840-853.

[7] D. Hefetz, M. Krivelevich, M. Stojaković and T. Szabó, Fast winning strategies in positional games, submitted. http://www.inf.ethz.ch/personal/szabo/PDF/fast.pdf.

[8] M. Stojaković, T. Szabó, Positional games on random graphs, Random Structures $\&$ Algorithms, 26 (2005) 204-223. 